\title{
Dividing attention between color and shape revisited: redundant targets coactivate only when parts of the same perceptual object
}

\author{
J. Toby Mordkoff • Rose Halterman Danek
}

Published online: 12 November 2010

(C) Psychonomic Society, Inc. 2010

\begin{abstract}
When a single perceptual object provides two different reasons for a particular decision (by containing two qualitatively different targets), detailed analyses of the response-time distributions have shown that the two different reasons are jointly responsible for the final decision. The question is whether this coactivation occurs because the two targets contained by the object were from separate dimensions (e.g., color and shape) or were parts of the same perceptual object. Early work argued in favor of dimensions, implying that the types of information being processed is critical, as opposed to their sources; more recent work has argued in favor of objects. Experiment 1 in the present paper corrected for a potential bias in the design of some recent studies and found additional evidence in favor of objects. Two additional experiments directly manipulated whether redundant targets would be perceived as parts of one or two perceptual objects (while holding all else constant) and produced the strongest evidence to date that coactivation requires that the redundant targets be parts of one object. This reverses the original conclusion and suggests that the sources of information are critical, as opposed to the types. Two specific versions of the objectbased model are discussed.
\end{abstract}

Keywords Divided attention · Perceptual dimensions .

Perceptual objects

People must often make rapid decisions in situations where there is more than one piece of relevant information. These

J. T. Mordkoff $(\bowtie) \cdot$ R. H. Danek

Department of Psychology, University of Iowa,

E11 Seashore Hall,

Iowa City, IA 52242, USA

e-mail: jonathan-mordkoff@uiowa.edu situations vary from the mundane, such as deciding between paper or plastic at the supermarket checkout (where both convenience and environmental impact are relevant), to the highly stressful, such as deciding whether to swerve to the left or the right when faced with a deer in the road (where both traction and on-coming traffic are relevant). One of the most basic questions that arise in the study of how rapid decisions are made is the manner in which multiple pieces of information are processed. Is all of the evidence in favor of a particular behavior combined in some way, or does one piece of evidence take near-total control, in something akin to a winner-take-all? Or it is not as simple as one or the other - does the manner in which multiple pieces of information are processed depend on the sources or the types of information? These questions can all be addressed by the use of the redundant-targets detection task (for an introduction, see Miller, 1982, or Mordkoff \& Yantis, 1991). The answers to these questions tell us much about the functional architecture of the human informationprocessing system, as well as how to design and build better devices to provide information to people who are faced with time-limited decisions.

Within the information-processing literature, the idea that multiple pieces of redundant information are somehow combined to activate a single decision is known as the coactivation model (e.g., Miller, 1982; Schwarz, 1989). The alternative idea, under which the strongest or fastest piece of information is wholly responsible for determining the response, is known as the race model (e.g., Raab, 1962). Both of these classes of model are consistent with the wellknown advantage for situations with more than one piece of evidence favoring the correct decision - a phenomenon known as a redundancy gain. The key question, therefore, when expressed in these terms, is under what conditions is each of these models correct. 
To date, three factors have been identified as determining whether a race or coactivation occurs. The first of these factors is the difficulty of the task: under simple reaction time, where any and all stimuli are targets and require the exact same response, coactivation appears to be the rule (see Giray \& Ulrich, 1993). In contrast, the evidence in favor of coactivation is much less consistent when the task requires a discrimination between targets and non-targets, as under go/no-go and forced choice (see Mordkoff, Miller, \& Roch, 1996).

The second factor is whether the experimental design as defined in terms of the frequencies of the various trial types - contains any biases in favor of redundant-target trials (see Mordkoff \& Yantis, 1991). If a go/no-go task is employed and no biased contingencies exist, then there is no evidence that identical visual targets coactivate responses; if the same task includes a bias that favors redundant-target trials, then evidence of coactivation can be observed. The only question here is whether this evidence should best be seen in terms of coactivation (since more than one stimulus is in some way responsible for behavior) or in terms of an interactive race between separate targets (Mordkoff \& Yantis, 1991). To side-step this issue, in what follows the phrase "evidence of coactivation" will be restricted to those situations where no such biases were present in the design.

The third factor that determines whether a race or coactivation occurs involves the specifics of the targets themselves. Because this is the question to be focused on here, it will be reviewed in much more detail. The first study to examine this issue directly (in a task requiring discriminations between targets and non-targets, and using a design with no biased contingencies) was that of Mordkoff and Yantis (1993). The working hypothesis was that separate decisions are made for each distal source of information. Therefore, separate pieces of evidence that derive from different sources would race to take control of behavior, whereas separate pieces of evidence that derive from the same source would coactivate. In contrast to the previous work that had failed to find consistent evidence of coactivation with identical targets (e.g., an $\boldsymbol{X}$ and an $\boldsymbol{X}$, where $X$ is the target), when the redundant targets were both features of a single object (e.g., a green $\boldsymbol{X}$, where both green and $X$ are targets), it was predicted that evidence of coactivation would now be observed.

The first experiment by Mordkoff and Yantis (1993) did, indeed, produce the predicted evidence of coactivation with conjoined shape and color targets (viz., the letters $\boldsymbol{X}, \boldsymbol{I}$, and $\boldsymbol{O}$ in green, cyan, and purple). This supported the working hypothesis that the source(s) of the information were critical. However, two follow-up experiments then suggested that the critical factor was the use of two different perceptual dimensions (i.e., one shape target and one color target), as opposed to the two targets being parts of one object. This alternative interpretation was supported by similar findings of evidence of coactivation when (1) the shape target was achromatic and surrounded by a colored frame and (2) the shape target was presented above fixation and the color target was below. If the distal-object sources were critical - or so it was argued - then separating the shape target from the color target should have eliminated the evidence of coactivation. The repeated finding of such evidence, even when the shape and color targets were in separate items on opposite sides of fixation, argued in favor of perceptual dimensions as the critical factor, instead.

In summary, until about 10 years ago, the general conclusion with regard to rapid, discriminative decisionmaking was that the manner in which multiple pieces of redundant information are processed depends on the types of information involved - with identical pieces of information racing to take control of behavior and qualitatively different pieces of information being somehow combined; the general conclusion was that the distal source(s) of the information was relatively unimportant. In terms of a concrete example, the conclusion was that the red color and the octagonal shape of a stop sign do not coactivate a rapid decision to apply the brakes because they are coming from the same distal object; they coactivate this decision because color and shape are qualitatively different.

\section{Objects vs. dimensions revisited}

More recently, however, the possibility that objects play the crucial role, as opposed to dimensions, has been reexamined by Feintuch and Cohen (2002). They suggested that it was possible for participants in all three of Mordkoff and Yantis' (1993) critical experiments to treat all elements of the displays as parts of a single object. For example, it might be the case that only when more than one item contains a task-relevant shape and/or more than one item contains a task-relevant color are the individual items within the displays actually processed as separate objects. When only one item has a task-relevant shape and only one item has a task-relevant color, then all items - regardless of spatial separation - can be treated as parts of a single, global object. Because all three of Mordkoff and Yantis' (1991) critical experiments involved only one task-relevant shape and only one task-relevant color in each of the displays, by this reasoning, both items could be treated as being parts of one object, such that an object-based model would be consistent with the evidence of coactivation. In short, the new idea was that the perceptual organization of the displays was actually responsible for the repeated evidence of coactivation, not the use of separable dimensions. 
To test this idea, Feintuch and Cohen (2002) conducted an experiment with two colored shapes in every display. (By the logic reviewed above, this would force participants to treat these two items as separate objects.) When they examined the redundant-target trials where both target features were parts of the same item - hereafter, "withinobject, redundant targets" - they found evidence of coactivation; when they examined the redundant-target trials where the shape target was part of one item and the color target was part of the opposite item - hereafter, "betweenobject, redundant targets" - they did not find such evidence. From this they concluded that objects, not dimensions, are the critical determinants of coactivation. In short: they reversed the conclusion of Mordkoff and Yantis (1993).

Unfortunately, the specific design that Feintuch and Cohen (2002) employed contained a subtle bias in favor of the within-object, redundant-target condition. (It's important to note that the potential for the sort of bias that was included in their design was not known at the time; it was first discussed in Mordkoff \& Halterman, 2008, which appeared several years later.) In brief, when both target features were parts of one item, only one possible combination of color and shape ever appeared in the opposite location. Even more, this particular combination of non-target color with non-target shape occurred either three or six times as often as the other specific color/shape conjunctions that were employed in the experiment. This occurred because the same combination of non-target color and non-target shape that was used as the second item in all within-object, redundant-targets displays was also included in every single-target display. In contrast, when the target color and target shape appeared as parts of different items, then, by definition, neither of the two items could be the high-frequency combination of non-target color and nontarget shape, since one item had to include the target color and the opposite item had to include the target shape.

The reason why this difference in the frequencies of specific color/shape conjunctions is important is that there is previous evidence that attention can be rapidly shifted away from any item (or attribute of an item) that is easily detected as being a non-target (see, e.g., Mordkoff, 1998, for a highly analogous case). Furthermore, it has recently been shown that these frequency- or contingency-based effects can be rooted in color/shape conjunctions, even when participants are instructed to ignore these particular items (Mordkoff \& Halterman, 2008). Therefore, the design used by Feintuch and Cohen (2002), with frequency ratios of three- and six-to-one, is potentially biased against the between-object, redundant-targets condition, since this was the only condition under which a rapid shift of attention away from the non-target item - and, presumably, therefore, towards the item containing the target feature(s) - would never occur.
Because of the central importance of which factor is responsible for the evidence of coactivation - viz., objects or dimensions - the present experiments were designed to re-examine this issue while removing all potential bias. Object-based models make the prediction that evidence of coactivation will consistently be found when the redundant target features are parts of the same perceptual object. In contrast, dimension-based models predict that evidence of coactivation will be found in any situation where one target is a color and the other is a shape, regardless of whether these features are parts of one or two objects. The former finding would support cognitive architectures under which decision-level processing is subject to constraints that are applied by perceptual organization. The latter finding would support architectures under which separate decisions are made within separable dimensions, regardless of perceptual organization. In other words, the results from these experiments should tell us which of two competing principles plays the greater role in rapid decision-making: consider the source (objects) or consider the argument (dimensions).

\section{Experiment 1}

The first experiment to be reported was a near-replication of Feintuch and Cohen's (2002) first experiment (i.e., two colored shapes in every display), but with the potential bias against between-object redundant targets removed. This was done by employing a wider variety of specific conjunctions of non-target color with non-target shape. Under the revised design, all specific color/shape conjunctions appeared equally often.

\section{Participants}

Twenty-four undergraduates were recruited from the Introductory Psychology Pool. All reported normal or corrected-to-normal, full-color vision. All were naïve as to the design and purpose of the experiment prior to their participation.

\section{Procedure and stimuli}

Participants began the experimental session with written instructions, followed by two practice blocks during which the color and shape targets were displayed for $5 \mathrm{~s}$ after an error. The remaining 16 blocks were retained for analysis and only used a brief tone to indicate that an error had been made (since errors after the first practice block were extremely rare). There was an enforced, 7-s break between blocks during which a summary of performance on the previous block was provided. 
Each trial began with the presentation of a white fixation cross (subtending $0.64^{\circ}$ of visual angle) for $500 \mathrm{~ms}$. After a $100-\mathrm{ms}$ blank, the final display was presented until a response was made or 1,000 ms elapsed. The final display contained two characters, each approximately $1.40^{\circ} \times$ $0.89^{\circ}$, with one item centered $1.53^{\circ}$ above fixation and the other $1.53^{\circ}$ below fixation. The inter-trial interval was $1,250 \mathrm{~ms}$.

There were approximately 40 trials in each block: the 36 planned trials, plus three, randomly-selected warm-up trials, plus a recovery trial after each error, plus the re-running of error trials at a random later point in the same block. Since participants rarely made more than one error in a block, most blocks included either 39 or 41 trials and required less than 2 min to complete.

Two sets of three shapes and three colors were employed. One set of shapes included the letters $\boldsymbol{A}, \boldsymbol{I}$, and $\boldsymbol{O}$; the second included $\boldsymbol{H}, \boldsymbol{M}$, and $\boldsymbol{S}$. One set of colors included blue, green, and red; the other included cyan, purple, and yellow. Each subject was randomly assigned one set of shapes and one set of colors as the "critical" features; the other set of shapes and the other set of colors acted as the "filler" features. (The use of this many different specific shapes and colors is required in order to eliminate any difference in the frequencies of specific combinations.) One shape from the critical set was selected to be the shape target; one color from the critical set was selected to be the color target. The task was go/no-go target detection: if either or both of the assigned targets were presented, a response was required; if neither target was presented, no response was to be made. Participants responded using the index finger on their favored hand.

\section{Designs}

The main design of the experiment was the same as that used by Mordkoff and Yantis (1993), which contains no biased contingencies between stimulus attributes or between nontarget attributes and whether a response should be made (see Mordkoff \& Yantis, 1991, for details on how to create an unbiased design). This core design has six types of trial that occur equally often: single-target shape, single-target color, redundant targets, and three different types of no-target trials that balance the three types of target-present trial. To be clear, assuming the critical shapes for a given subject were $\boldsymbol{A}, \boldsymbol{I}$, and $\boldsymbol{O}$ as the target, distractor, and noise values, respectively, and the critical colors for the subject were blue, green, and red as target, distractor, and noise, then the following six pairs would all be used equally often: blue \& $\boldsymbol{A}$, blue $\& \boldsymbol{I}$, green $\& \boldsymbol{A}$, red $\& \boldsymbol{I}$, green $\& \boldsymbol{O}$, and red $\& \boldsymbol{O}$. (The distinction between distractor and noise values is that distractors sometimes appear with targets, while noise values never appear with targets. This distinc- tion is required to produce an unbiased design; see Mordkoff \& Yantis, 1991.) Note how one third of these pairs contains each of the three colors and one third contains each of the shapes. Balancing this, only six combinations of the filler colors and shapes were used to complete the displays, and these, also, were evenly divided across the three filler colors and the three filler shapes. An example set of filler features to go with the critical items listed above are: cyan \& $\boldsymbol{H}$, cyan \& $\boldsymbol{M}$, purple \& $\boldsymbol{H}$, yellow \& $\boldsymbol{M}$, purple \& $\boldsymbol{S}$, and yellow \& $\boldsymbol{S}$.

In order to also include a manipulation of whether the two target features on redundant-targets trials occurred in same or different items, as well as to balance the frequencies at which targets appeared in the upper and lower locations, one quarter of the trials placed both critical features in the upper location, one quarter placed both in the lower location, and the remaining half placed one critical feature in the upper location and the other critical feature in the lower location. Using the example from the previous paragraph (with blue and $\boldsymbol{A}$ being the targets), this implies that one-quarter of the redundant-target displays included a blue $\boldsymbol{A}$ in the upper location, one-quarter included a blue $\boldsymbol{A}$ in the lower location, one-quarter included a blue non- $\boldsymbol{A}$ in the upper location and a non-blue $\boldsymbol{A}$ in the lower location, and the final quarter included a non-blue $\boldsymbol{A}$ in the upper location and a blue non- $\boldsymbol{A}$ in the lower location. The specific pair of filler color and filler shape that was used to complete a given display was selected at random, subject to the limitation that only six of the nine possible combinations would ever be used (see above).

\section{Method of analysis}

The initial analysis merely concerned mean correctresponse RT as a function of condition (i.e., the standard tests for redundancy gains in mean RT), done separately for the within- and between-object redundant-target trials. The more detailed analysis employed the race-model inequality (RMI; Miller, 1982), looking for evidence of coactivation (see, e.g., Mordkoff \& Yantis, 1991, 1993, for details on how to conduct these tests). In brief, evidence of coactivation is provided when the following equation is violated, since this equation places an upper bound on the cumulative distribution of redundant-targets RTs according to all separate-activations models:

$\mathrm{p}(\mathrm{RT}<\mathrm{t} \mid \mathrm{Ts} \& \mathrm{Tc}) \leq \mathrm{p}(\mathrm{RT}<\mathrm{t} \mid \mathrm{Ts})+\mathrm{p}(\mathrm{RT}<\mathrm{t} \mid \mathrm{Tc})$

where $\boldsymbol{T} \boldsymbol{s}$ and $\boldsymbol{T c}$ stand for the target shape and color, respectively, such that $\boldsymbol{T} \boldsymbol{s} \& \boldsymbol{T} \boldsymbol{c}$ represents a redundanttargets trial, $\boldsymbol{T} \boldsymbol{s}$ represents a single-target shape trial, and $\boldsymbol{T} \boldsymbol{c}$ represents a single-target color trial. The RMI requires that the probability of responding by any time $t$ given redundant 
targets be less than or equal to the sum of the probabilities of responding by that same time $t$ given only a shape target or only a color target. All separate-activations models, including the standard race model, must obey this rule. In contrast, coactivation models are consistent with violations of this rule, and the best-known version of this type of model actually predicts violations under most conditions (see Schwarz, 1989).

Technical note While the above is an accurate description of the RMI as it applies in theory to the results from these experiments, it is not how the data are actually analyzed (see, e.g., Miller, 1982; Mordkoff \& Yantis, 1991, for additional details). Instead, by capitalizing on the monotonic/non-decreasing nature of cumulative distribution functions, the reliability of any violation of the RMI is in practice evaluated in the following manner: (1) for each participant separately, the values of RT that correspond to the 5th through 95th percentiles (at 5\% intervals) are found for the redundant-targets condition; (2) for each participant, the values of RT for which the sum of the percentiles for the two single-target conditions equals $5 \%$ through $95 \%$ are also found; finally, (3) a paired-samples t-test is conducted at each percentile where the mean value of RT (across participants) for the redundant-targets condition is lower than that for the sum of the single-target conditions, since these differences in favor of redundant-target trials constitute mean violations of the RMI. The reason for evaluating the RMI in terms of RT, instead of probability, is that this allows the data to be Vincentized across participants, preserving the shape of the individual distributions of RT (see Thomas \& Ross, 1980). If this approach is not taken (i.e., if the data are analyzed in terms of probabilities, instead of values of time), then only the fastest participants would contribute to most of the critical tests, since only the fastest participants would have probabilities above zero for smallest values of $t$ in the original equation. Finally, because the finding of a slower RT for the redundant-targets condition is not informative (since the race-model inequality is one-sided), t-tests are not performed at percentiles that do not exhibit a positive violation.

\section{Results}

The mean correct RTs were $378.6 \mathrm{~ms}$ and $388.5 \mathrm{~ms}$ in the color- and shape-target only conditions; and $331.1 \mathrm{~ms}$ and $353.1 \mathrm{~ms}$ in the within-object and between-objects redundant-targets conditions, respectively. The redundancy gain of $52.5 \mathrm{~ms}$ for the within-object condition was reliable; $\mathrm{t}(23)=14.01, p<.001$. The redundancy gain of $30.4 \mathrm{~ms}$ for the between-objects condition was also reliable; $\mathrm{t}(23)=$ $9.28, p<.001$. Errors were too infrequent to analyze; participants failed to respond on less than $0.5 \%$ of the target-present trials and made false-alarm errors on only $2.24 \%$ of the target-absent trials.

The mean violations of the RMI are displayed in Fig. 1. As can be seen, for the comparisons involving withinobject redundant-target trials there was a wide range of percentiles for which the RMI was violated. All of the mean violations from the 5 th to the 60 th percentiles were reliable; all $\mathrm{t}(23) \geq 2.16, p<.05$. In contrast, there was only one mean violation of the RMI (of less than $1 \mathrm{~ms}$ ) for the comparisons involving the between-objects trials, and it was not reliable; $\mathrm{t}(23)=0.31$.

\section{Discussion}

The results from Experiment 1 provide prima facie evidence that objects, as opposed to dimensions, are critical to whether evidence of coactivation will be observed. When the redundant targets were both parts of one display item, numerous violations of the RMI were found. In contrast, when the redundant targets were parts of different items, the RMI was consistently obeyed. These findings replicate those of Feintuch and Cohen (2002), extending the pattern of results to a situation without any potential bias from unbalanced display frequencies.

Even more, these data argue quite strongly against the model that Mordkoff and Yantis (1993) presented in their Discussion. That model placed all of the stress on perceptual dimensions and did not include an object-based component. Without drastic modification, therefore, that model would predict evidence of coactivation in both of the present comparisons, since both the within- and betweenobject conditions involved targets from the separable dimensions of shape and color.

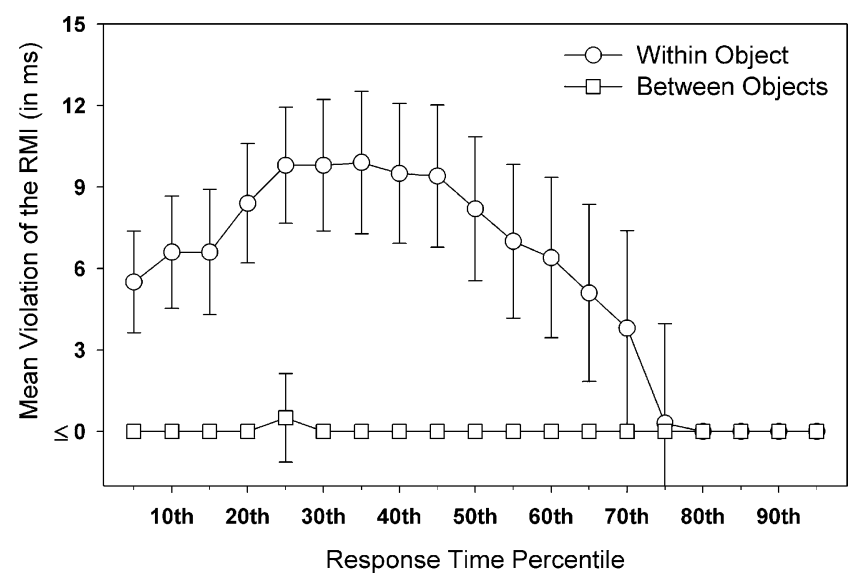

Fig. 1 Race-model inequality violations from Experiment 1. (For clarity, error bars are only included when the data exhibit a mean violation of the RMI. As can be seen, only the within-object condition produced significant evidence of coactivation) 


\section{Experiment 2}

The results from Experiment 1 provided evidence of coactivation when the two target features were parts of the same display item, but not when the two target features were parts of different items. There are two reasons, however, why this fails to convincingly demonstrate that coactivation only occurs within objects. First, there is the confound between whether the two targets were parts of the same item and whether the two targets appeared in the same, small, and well-defined spatial area (see, e.g., Duncan, 1984, for a cogent discussion of this particular problem). Second, no attempt was made to directly manipulate whether the two target features were actually perceived as being parts of the same or different objects.

Experiment 2 deals with both of these weaknesses by capitalizing on a well-established procedure for controlling whether separate display items are grouped as parts of the same object or segregated as parts of different objects: viz., the Gestalt principle of common fate (see, e.g., Driver \& Baylis, 1989, for a previous use of this technique in an information-processing task). To do this, Experiment 2 returned to the basic method employed in the second experiment of Mordkoff and Yantis (1993) under which an achromatic shape was surrounded by a colored frame. The main change in method was that, in the present experiment, the two items did not remain stationary. Instead, on half of the trials - hereafter, the "unified-motion" condition - the two items oscillated together between two locations on opposite sides of fixation. On the other half of the trials the "separate-motions" condition - the achromatic shape oscillated between one pair of locations, while the colored frame oscillated between a different pair of locations. The former condition produced a strong impression of a single object; the latter produced an equally strong impression of two separate objects.

\section{Participants}

Twenty-four new undergraduates were recruited from the same population as Experiment 1.

\section{Procedure and stimuli}

The general procedure was the same as for Experiment 1 (including the number of task, blocks, trials per block, practice, instructions, and feedback, etc.). The crucial difference is that the final displays in this experiment contained a single, white letter $\left(1.40^{\circ} \times 0.89^{\circ}\right)$ and a larger, colored frame $\left(2.10^{\circ} \times 1.34^{\circ}\right)$, instead of two colored letters. Each of these two items shifted back-and-forth between two locations (separated by $1.90^{\circ}$ ) every $100 \mathrm{~ms}$ with no inter-stimulus interval. The two locations were equally distant from fixation in exactly opposite directions. The specific direction(s) away from fixation was selected at random on every trial. Thus, for example, on one trial the white letter might oscillate between locations that correspond to 1 and 7 o'clock; on the next trial it might oscillate between 3 and 9 o'clock. The key manipulation - besides the inclusion of varying numbers of target features - was whether, on a given trial, the white letter and colored frame oscillated between the same or different pairs of locations.

In the unified-motion condition, the white letter and colored frame used the same pair of alternating locations such that the two items "jumped" back and forth across fixation as one. In the separate-motions condition, a pair of locations was first selected at random for the white letter, and then a second pair was calculated for the colored frame such that the latter's motion was exactly orthogonal. For example, if the white letter was determined to oscillate between 2 and 8 o'clock, then, assuming the separate-motions conditions, the colored frame would oscillate between 5 and 11 o'clock. The two items continued to oscillate until a response was made or 1,000 ms had elapsed and the trial was terminated, and a no-go response was recorded.

Because the displays in this experiment contained only one white letter and a colored frame (as opposed to two colored letters, as in Experiment 1), only one set of three letters $(\boldsymbol{A}, \boldsymbol{I}$, and $\boldsymbol{O}$, or $\boldsymbol{H}, \boldsymbol{M}$, and $\boldsymbol{S})$ and one set of three colors (blue, green, and red, or cyan, purple, and yellow) were required. Which sets were used was counter-balanced across participants.

\section{Designs and analysis}

As in Experiment 1, the core design of this experiment was identical to that used by Mordkoff and Yantis (1993), under which six different, basic displays occur equally often. Also as before, the core design was exactly divided by two, such that half of the trials involved unified motion and the other half involved separate motions. Thus, whether the items moved together or orthogonally was unrelated to whether (or which) targets were shown.

The analysis was the same manner as for Experiment 1, with separate tests for redundancy gains and violations of the RMI being conducted for the unified- and separate-motions conditions. A preliminary test found no differences between unified- and separate-motions trials when only one target was included, so these data were treated as interchangeable. Only the redundant-targets data depended on and, therefore, were kept separate as a function of motion condition.

Results and discussion

The mean correct RTs were $288.1 \mathrm{~ms}$ and $304.0 \mathrm{~ms}$ in the color and shape single-target conditions, and $257.5 \mathrm{~ms}$ and 
$261.9 \mathrm{~ms}$ in the unified- and separate-motions redundanttargets conditions, respectively. Both redundancy gains were reliable: $\mathrm{t}(23)=14.50, p<.001$, for unified motion; $\mathrm{t}(23)=$ $9.68, p<.001$, for separate motions. Errors, again, were too infrequent to analyze; the miss rate was $0.17 \%$, and the false-alarm rate was $2.22 \%$.

The mean violations of the RMI are shown in Fig. 2. For the comparisons involving the unified-motion redundanttarget trials, the violations at the 5th through 25 th percentiles were reliable; all $\mathrm{t}(23) \geq 1.92, p<.05$. The single mean violation of the RMI (of less than $1 \mathrm{~ms}$ ) for the comparisons involving the separate-motions data was not reliable; $\mathrm{t}(23)=0.28$.

These data confirm and extend the findings from Experiment 1: when two redundant targets are both parts of the same perceptual object, as defined by common fate, strong evidence of coactivation is found; when the same two redundant targets are parts of separate objects, as defined by orthogonal motion, no significant evidence of coactivation is found.

\section{Experiment 3}

One criticism that could be leveled at the method used in Experiment 2 (in an attempt to undermine the evidence in favor of the crucial role of perceptual objects) is that the displays employed in the unified-motion and separatemotions conditions were noticeably different in several respects. Most of all, in the unified-motion condition, the achromatic shape and colored frame were always centered on the same location (at any given time), so the contours of each never crossed or overlapped. In contrast, in the separate-motions condition, the two items often overlapped and/or partially occluded each other, which could have

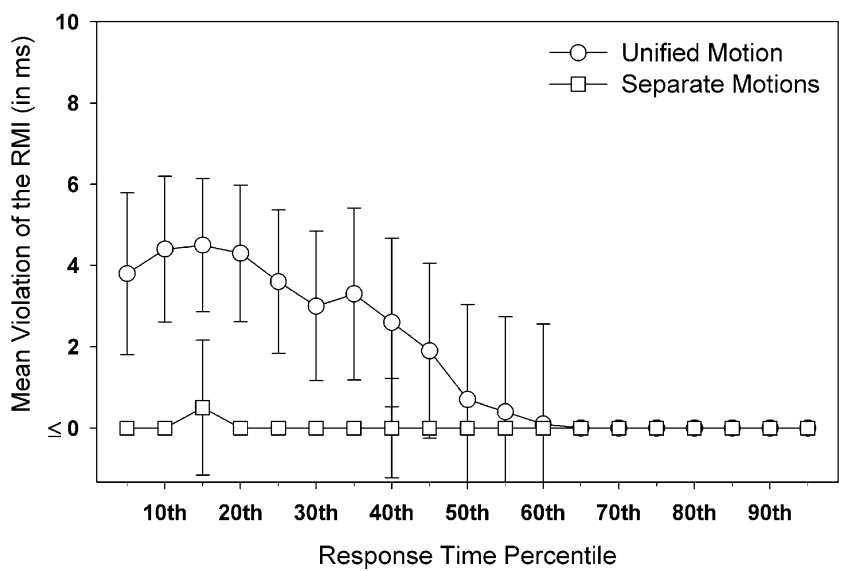

Fig. 2 Race-model inequality violations from Experiment 2. (Error bars are only included when the data exhibit a mean violation of the RMI. As can be seen, only the unified-motion condition produced significant evidence of coactivation) altered perceptual processing in such a way as to prevent any evidence of coactivation.

To control for this possibility, Experiment 3 was designed to be even more similar to the second experiment of Mordkoff and Yantis (1993), where the achromatic shape and colored frame were always both centered on fixation. In order to induce the perception of one or two objects, prior to the appearance of the critical features, the motion histories of the two items were manipulated (see, e.g., Moore \& Lleras, 2005, for a previous example). In the new versions of the unified-motion and separate-motions conditions, placeholders for the achromatic shape and colored frame oscillated in the same manner as used in Experiment 2 - either together or at right angles to each other. In contrast to Experiment 2, however, during the time that these oscillations occurred - hereafter, the "motion-history period" - neither the critical shape nor the critical color was actually present. The shape placeholder only switched to being a letter (by deleting two bars from a block figureeight), and the frame placeholder only switched to being colored (from an equiluminant gray), at the end of the motion-history period, when the two items were both finally centered on fixation and all motion ceased.

Technical note In an unreported experiment, at the end of the motion-history period, when the shape and frame placeholders switched to being critical features, the final display remained visible until a response was made or $1,000 \mathrm{~ms}$ had elapsed (and the trial was coded as being a "no-go"). This procedure, however, failed to produce any differences between the unified-motion and separatemotions conditions (i.e., both produced evidence of coactivation, matching the findings of Mordkoff \& Yantis, 1993). This seems to have occurred because the static presence of the achromatic shape and colored frame, for hundreds of milliseconds, was sufficient to override the motion-history manipulation, causing both conditions to effectively be the same as that used previously. To avoid this problem, in the present Experiment 3, the final display on each trial (when the critical letter and colored frame were actually shown) was only presented for the same duration as each of the oscillating placeholder displays. As will be seen, this method succeeded in allowing the motionhistory manipulation to be quite effective.

\section{Method}

This experiment differed from the previous in the following way: the unified- vs. separate-motions manipulation was implemented prior to the appearance of the final display (as opposed to being alternative methods of creating the final displays). Each trial began with the appearance of a block, grey figure- 8 , and a larger grey frame (the same sizes as the 
letters and frames in Experiment 2). These either oscillated together or orthogonally in the same manner as used in Experiment 2 for a total of $600 \mathrm{~ms}$. Finally, two bars from the block figure-8 were deleted to "reveal" one of three letters $(\boldsymbol{H}, \boldsymbol{P}$, or $\boldsymbol{S})$, and the frame switched to being one of three colors (either blue, green, or red; or cyan, purple, or yellow; counter-balanced across participants). This final display was presented for only $100 \mathrm{~ms}$ (for reasons mentioned above)

Most important: the grey letter and colored frame in the final display were always both centered on fixation, regardless of motion-history condition, such that the only display that contained the critical color and shape for the trial was identical across conditions (and almost identical to the final display in Experiment 2 of Mordkoff \& Yantis, 1993). Expressed differently: if the motions of the figure8 and grey-frame placeholders are ignored, then there is no difference at all between the unified- and separate-motions conditions of this experiment.

\section{Results and discussion}

The mean RTs were $324.9 \mathrm{~ms}$ and $350.3 \mathrm{~ms}$ in the colorand shape-target only conditions. This difference was reliable: $\mathrm{t}(23)=5.56, p<.001$. For redundant targets, the mean RTs were $294.7 \mathrm{~ms}$ and $298.7 \mathrm{~ms}$ in the unified- and separate-motions conditions. Both redundancy gains were reliable: $\mathrm{t}(23)=20.39, p<.001$, for unified motion; $\mathrm{t}(23)=$ $24.39, p<.001$, for separate motions. Errors, as before, were highly infrequent, but did differ as a function of number of targets: $0.50 \%$ vs. $0.35 \%$ for single- vs redundant-target trials, $\mathrm{t}(23)=1.82, p<.05$. The false-alarm rate was $2.71 \%$.

The mean violations of the RMI are shown in Fig. 3. In the case of unified motion, the violations at the 20th through 35 th percentiles were reliable; all $\mathrm{t}(23) \geq 1.93, p<.05$. In the

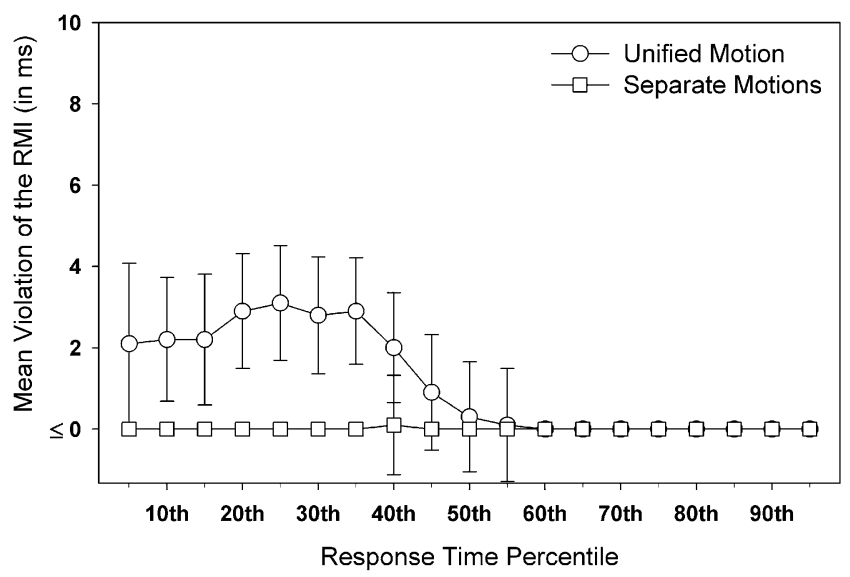

Fig. 3 Race-model inequality violations from Experiment 3. (Error bars are only included when the data exhibit a mean violation of the RMI. As can be seen, only the unified-motion condition produced significant evidence of coactivation) case of separate motions, there was a single mean violation of the RMI (of $0.1 \mathrm{~ms}$ ) at the 40th percentile; $\mathrm{t}(23)=0.02$. In a sentence: even when the difference between conditions is established by history and no differences exist in the critical displays, evidence of coactivation depends on whether the redundant targets are parts of the same perceptual object.

\section{General discussion}

The present work re-examined the question of whether redundant pieces of information are processed separately or are combined and coactivate a single, rapid decision. Mordkoff and Yantis (1993) originally hypothesized that the critical issue would be the source(s) of the pieces of information - that coactivation would only occur when redundant target features are parts of the same perceptual object. Their data, however, suggested that the critical factor was the type of information, instead - that coactivation occurs when redundant targets are qualitatively different from each other, such as one being a color and the other being a shape. This conclusion was later criticized by Feintuch and Cohen (2002), who pointed out that the specific displays employed by Mordkoff and Yantis (1993) may have always evoked the percept of a single object (and not two, as had been assumed). In light of this weakness in the previous research, and given the importance of the question of whether information is organized in terms of source (object) or type (dimension), we here re-examined this question and found much stronger evidence in favor of objects. In summary, it now appears that coactivation occurs under at least three sets of conditions: (1) when a simple RT task is employed (Giray \& Ulrich, 1993), (2) when the experimental design contains biased contingencies in favor of redundant-targets trials (Mordkoff \& Yantis, 1991), and (3) when the redundant targets are parts of the same perceptual object. There is no remaining evidence to suggest that the use of separable dimensions is in any way critical, except to the extent that using separable dimensions is the easiest way to include redundant target features in one perceptual object.

Before going on to discuss some possible, specific explanations for this pattern of results, it is important to note that the present work does not rule out some remaining, non-trivial role for separable dimensions and/ or other display attributes, such as spatial location. First, whereas being perceived as parts of the same perceptual object appears to be necessary for coactivation, it is not always sufficient. When targets from the same dimension are grouped in some ancillary manner (e.g., being surrounded by a common frame), they can still fail to produce evidence of coactivation, even when targets from different dimensions - presented under the same conditions - 
do produce evidence of coactivation (see, e.g., Feintuch \& Cohen, 2002, Expts 2 \& 3). Even stronger: when redundant simple targets are presented to different ears, if the targets are identical, such they are perceived as constituting a single stimulus, then not only is no evidence of coactivation observed, but even the basic redundancy gain in mean RT can be lost (e.g., Schröter, Ulrich, \& Miller, 2007). Thus, in order to coactivate, redundant targets need to be parts of the same perceptual object while still being different enough to be processed as two different pieces of information. And one of the easiest ways to keep redundant targets perceptually separate is to employ targets from separable dimensions.

Second, in pop-out search tasks, the magnitude and extent of coactivation has been found to depend on the spatial separation between the two targets (see, e.g., Krummenacher, Müller, \& Heller, 2002a, 2002b). Redundant targets that are closer together produce stronger evidence of coactivation than redundant targets that are widely separated. In this case, the explanation centers on the frequency (across trials) at which the two targets are successfully grouped. Pop-out targets that are nearer to each other will more often be grouped as one perceptual object and, therefore, coactivate on a larger percent of the trials. Thus, pop-out targets that are closer to each other produce stronger evidence of coactivation.

Returning, now, to the specific mechanisms that might underlie the critical role of objects in determining whether coactivation occurs, we see two classes of explanation for the entire pattern of results to date. The first class of models posits a direct effect of perceptual organization due to the underlying mental architecture (see, e.g., the Introduction of Mordkoff \& Yantis, 1993). On this view, separate decisions with regard to target presence are made for each perceptual object. Within a given object, all evidence in favor of a given response is pooled. Therefore, coactivation occurs when redundant targets are parts of the same object because, for example, these targets are processed by the same cognitive unit or module. In contrast, because separate decisions are made for each object, when redundant targets are parts of separate objects they cannot coactivate; instead, they can only participate in a race (between modules) to trigger the response.

The second class of models posits an indirect effect of perceptual organization, with visual attention acting as the mediator (see, e.g., Feintuch \& Cohen, 2002). For example, the information-processing architecture might be arranged such that redundant targets (of any type, from any source) always have the opportunity to coactivate a response. In other words, all evidence in favor of a given response is always potentially pooled or summed by a single decision mechanism, regardless of the source of the evidence. However, coactivation can only actually occur when the redundant targets finish processing at approximately the same time; if the redundant targets finish processing at highly different times, then pooling becomes irrelevant because the response will have already been triggered by the time the second target's activation arrives at the point of summation (see Miller, 1986, for an extended discussion of this issue and examples of evidence). On the additional assumption that visual attention is allocated in terms of perceptual objects (see, e.g., Bundesen, 1990; Duncan, 1984; Vecera \& Farah, 1994), then redundant targets that are parts of the same object are much more likely to finish processing simultaneously than are redundant targets from different objects. This holds because (1) processing speed depends on the amount of attention, and (2) features of the same object receive the same amount of attention, whereas features of separate objects receive opposite amounts of attention. Therefore, even though the potential for coactivation is always present (according to this view), violations of the race-model inequality are only found in those cases where the redundant target features are parts of the same perceptual object due to the mediating influence of visual attention.

The distinction between these two classes of objectbased model may appear to be subtle, but should still be investigated in future research. Most of all, the difference between these models goes to the heart of the question that is addressed by this line of research: whether rapid decisions are always based on all of the (currently) available information or whether, in some cases, one or more pieces of relevant information are, for some reason, unfortunately, sadly, ignored.

Author Note The authors thank the reviewers and editor for their helpful comments.

\section{References}

Bundesen, C. (1990). A theory of visual attention. Psychological Review, 97, 523-547.

Driver, J., \& Baylis, G. C. (1989). Movement and visual attention: The spotlight metaphor breaks down. Journal of Experimental Psychology: Human Perception and Performance, 15, 448-456.

Duncan, J. (1984). Selective attention and the organization of visual information. Journal of Experimental Psychology: General, 113, 501-517.

Feintuch, U., \& Cohen, A. (2002). Visual attention and coactivation of response decisions for features from different dimensions. Psychological Science, 13, 361-369.

Giray, M., \& Ulrich, R. (1993). Motor coactivation revealed by response force in divided and focused attention. Journal of Experimental Psychology: Human Perception and Performance, $19,1278-1291$.

Krummenacher, J., Müller, H. J., \& Heller, D. (2002a). Visual search for dimensionally redundant pop-out targets: Redundancy gains in compound tasks. Visual Cognition, 9, 801-837.

Krummenacher, J., Müller, H. J., \& Heller, D. (2002b). Visual search for dimensionally redundant pop-out targets: Parallel-coactive 
processing of dimensions is locations specific. Journal of Experimental Psychology: Human Perception and Performance, 28, 1303-1322.

Miller, J. (1982). Divided attention: Evidence for coactivation with redundant signals. Cognitive Psychology, 14, 247-279.

Miller, J. (1986). Timecourse of coactivation in bimodal divided attention. Perception \& Psychophysics, 40, 331-343.

Moore, C. M., \& Lleras, A. (2005). On the role of object representations in substitution masking. Journal of Experimental Psychology: Human Perception and Performance, 31, 11711180.

Mordkoff, J. T. (1998). Between dimension flanker effects: A clarification with encouraging implications. Psychonomic Bulletin \& Review, 5, 670-675.

Mordkoff, J. T., \& Halterman, R. (2008). Feature integration without visual attention: Evidence from the correlated flankers task. Psychonomic Bulletin \& Review, 15, 385-389.

Mordkoff, J. T., \& Yantis, S. (1991). An interactive race model of divided attention. Journal of Experimental Psychology: Human Perception and Performance, 17, 520-538.
Mordkoff, J. T., \& Yantis, S. (1993). Dividing attention between color and shape: Evidence of coactivation. Perception \& Psychophysics, 53, 357-366.

Mordkoff, J. T., Miller, J., \& Roch, A.-C. (1996). Absence of coactivation within the motor component: Evidence from psychophysiological measures of target detection. Journal of Experimental Psychology: Human Perception and Performance, 22, 25-41.

Raab, D. (1962). Statistical facilitation of simple reaction time. Transactions of the New York Academy of Sciences, 43, 607-610.

Schröter, H., Ulrich, R., \& Miller, J. O. (2007). Effects of redundant auditory stimuli on reaction time. Psychonomic Bulletin \& Review, 14, 39-44.

Schwarz, W. (1989). A new model to explain the redundant signals effect. Perception \& Psychophysics, 46, 498-500.

Thomas, E. A. C., \& Ross, B. H. (1980). On appropriate procedures for combining probability distributions within the same family. Journal of Mathematical Psychology, 21, 136-152.

Vecera, S. P., \& Farah, M. J. (1994). Does visual attention select objects or locations? Journal of Experimental Psychology: General, 123, 146-160. 